\title{
ARTICLE
}

Received 9 Feb 2010 | Accepted 30 Jun 2010 | Published 27 Jul 2010

DOI: $10.1038 /$ ncomms1045

\section{Ancestry of motor innervation to pectoral fin and forelimb}

\author{
Leung-Hang Ma ${ }^{1,2}$, Edwin Gilland ${ }^{2,3}$, Andrew H. Bass ${ }^{2,4}$ \& Robert Baker ${ }^{1,2}$
}

Motor innervation to the tetrapod forelimb and fish pectoral fin is assumed to share a conserved spinal cord origin, despite major structural and functional innovations of the appendage during the vertebrate water-to-land transition. In this paper, we present anatomical and embryological evidence showing that pectoral motoneurons also originate in the hindbrain among ray-finned fish. New and previous data for lobe-finned fish, a group that includes tetrapods, and more basal cartilaginous fish showed pectoral innervation that was consistent with a hindbrain-spinal origin of motoneurons. Together, these findings support a hindbrain-spinal phenotype as the ancestral vertebrate condition that originated as a postural adaptation for pectoral control of head orientation. A phylogenetic analysis indicated that Hox gene modules were shared in fish and tetrapod pectoral systems. We propose that evolutionary shifts in Hox gene expression along the body axis provided a transcriptional mechanism allowing eventual decoupling of pectoral motoneurons from the hindbrain much like their target appendage gained independence from the head.

\footnotetext{
1 Department of Physiology and Neuroscience, NYU Langone Medical Center, New York, New York 10016, USA. ${ }^{2}$ Marine Biological Laboratory, Woods Hole, Massachusetts 02543, USA. ${ }^{3}$ Department of Anatomy, Howard University College of Medicine, Washington, District of Columbia 20059, USA. ${ }^{4}$ Department of Neurobiology and Behavior, Cornell University, Ithaca, New York 14853, USA. Correspondence and requests for materials should be addressed to R.B. (email: Robert.baker@nyumc.org).
} 
Phavioural innovations associated with vertebrate locomotion have depended on functional transformations of the anterior paired appendages. These range from the pectoral fin's role in hydrodynamic mechanisms of balance and swimming to the forelimb's contribution to weight-bearing locomotion ${ }^{1}$. Although the ancestry of these appendages has received much attention ${ }^{2,3}$, the evolutionary origin of the corresponding motor circuitry remains largely unexplored. The spinal cord position of tetrapod forelimb circuitry is often presented as the ancestral condition of vertebrates and is presumed to be the location for the corresponding pectoral circuit in bony fish ${ }^{4-7}$. However, analyses of fish pectoral nerves suggest an alternative organization. Among both main clades of bony vertebrates, sarcopterygian (lobe-finned) and actinopterygian (ray-finned) fish ${ }^{8}$, adult pectoral fins are innervated by spinal nerves, as well as by nerves that exit the posterior, occipital region of the skull ${ }^{9-12}$. This innervation pattern suggests that pectoral fin motoneurons might originate from both the brain and spinal cord instead of from just the spinal cord. However, nerve trajectory alone cannot be used to identify the ancestral pattern of motor innervation to pectoral appendages because fusion of anterior vertebrae with the occiput during growth can substantially modify the occipital skull ${ }^{13}$. To directly test the hypothesis that pectoral motoneurons have evolutionary and developmental origins from both the hindbrain and spinal cord, we mapped their location in the developing neuroepithelium of actinopterygian fish.

Actinopterygians were the main focus of our investigation because, unlike sarcopterygians, the embryos of several species were readily available for the comparative anatomical and molecular analyses essential to determining the ancestry of pectoral neurons and innervation. We included a basal actinopterygian, the acipenseriform Polydon spathula (paddlefish), and several species from the more derived, species-rich group of teleosts ${ }^{8}$. The teleosts chosen were five key study species from three distantly related groups, widely investigated at molecular, neural and behavioural levels of analysis ${ }^{14}$ : cypriniforms (zebrafish Danio rerio, goldfish Carassius auratus), salmoniforms (salmon Salmo salar, trout Salvelinus fontinalis) and batrachoidiforms (midshipman Porichthys notatus). Zebrafish included a hoxb4a enhancer trap line ${ }^{15}$ expressing a genetic marker demarcating the hindbrain-spinal cord boundary ${ }^{16}$. Using this comparative developmental strategy, we precisely aligned the axial position of developing pectoral motoneurons with other brain nuclei, myotomes and peripheral nerves. We demonstrate that pectoral motoneurons exhibit a conserved pattern of dual origin in both the hindbrain and spinal cord with no migration throughout development. Together with new and previous evidence for occipital and spinal innervation of pectoral fins in sarcopterygian (lungfishes and coelacanth) and more basal cartilaginous (chimaera) fish, the results indicate that this pattern of pectoral innervation is the primitive condition for jawed vertebrates. Pectoral motor control centred at a hindbrainspinal location was likely a novel adaptation for head orientation and swimming manoeuvres via the occipital-pectoral skeletal connection in primitive fish, including those that gave rise to tetrapods.

\section{Results}

Location of embryonic pectoral motoneurons. The pectoral fin is innervated by a plexus of nerves, members of which are designated as either occipital $(\mathrm{Oc})$ or spinal $(\mathrm{Sp})$ depending on the location of their roots relative to the cranio-vertebral interface ${ }^{9-12}$. In all four actinopterygians studied, including midshipman (Fig. 1a) and zebrafish (Fig. 1b), the anterior most pectoral nerve emerged from the skull through an occipital foramen or the foramen magnum (for example, Fig. 1c,d; Supplementary Figs S1-S2). Although the innervation pattern was grossly conserved, the substantial modifications in the occipital skull produced by its fusion with parts of anterior vertebrae ${ }^{13}$ precluded a conclusive comparison between species. We found, however, that alignment of the fin bud, myotomes and neuroepithelium was strikingly similar at early fin bud stages of development across all four species (Fig. 1e; Supplementary Fig. S3). This finding allowed us to study pectoral innervation at comparable embryonic stages across taxa, independent of speciesspecific skeletal modifications during subsequent growth.

Pectoral motoneurons were visualized by injecting a saturated solution of fluorescent lipophilic dye (DiD) into the fin bud that was incorporated into individual axons and diffused along the membrane to label the cell body. Subsequent confocal imaging revealed a consistent dual hindbrain-spinal origin of pectoral motoneurons in actinopterygians. Embryonic pectoral motor nuclei always extended as a column between myotomes (M) 2-3 and 5-6, with separate nerve roots projecting through each successive myotome, starting invariantly with
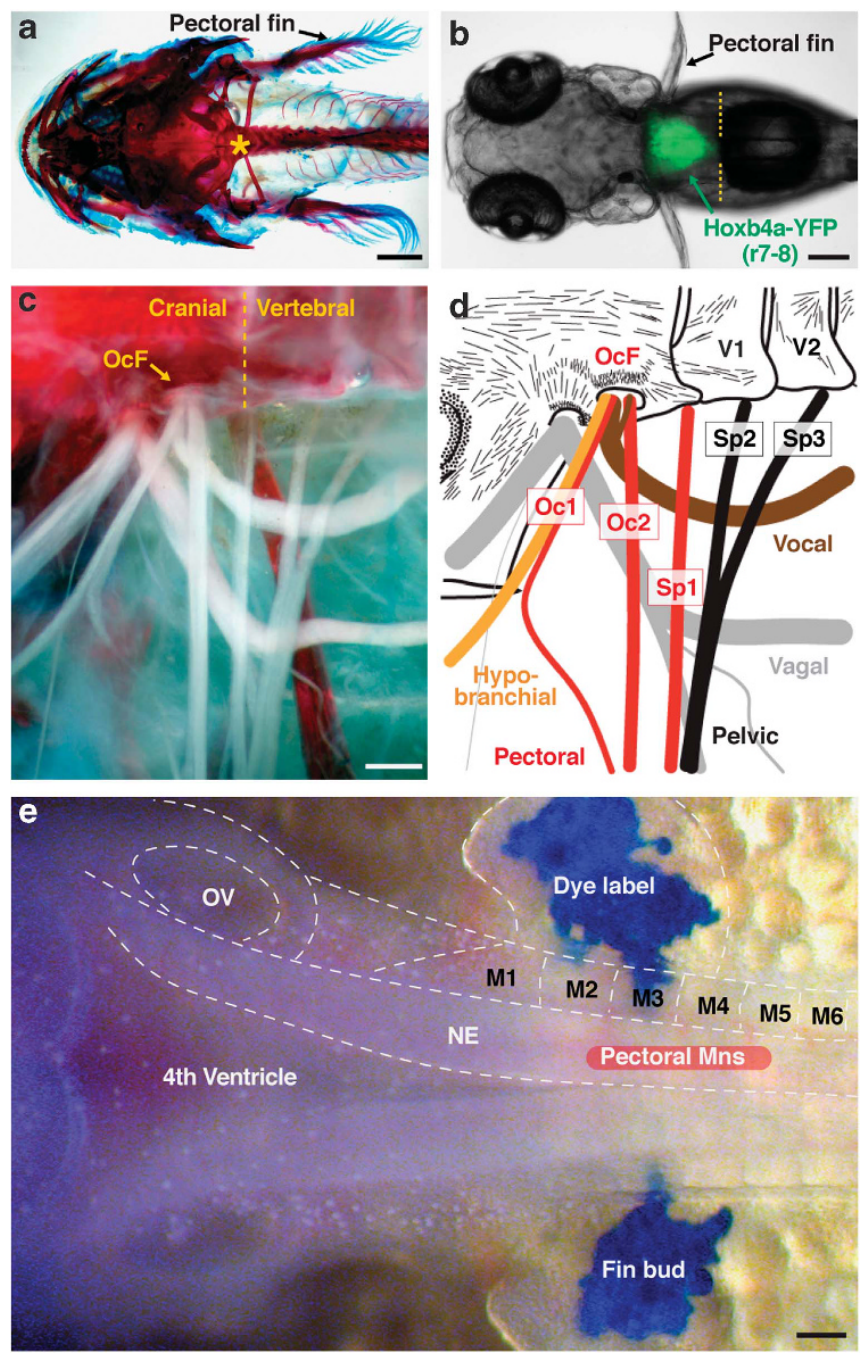

Figure 1 | Occipital region in actinopterygian fish. (a) Cranio-vertebral junction (asterisk) in a juvenile midshipman stained with alcian blue and alizarin red. (b) Hindbrain-spinal cord boundary (yellow hatching) demarcated in the zebrafish hoxb4a enhancer trap line. (c) Pectoral fin innervation in juvenile midshipman. (d) Schematic drawing of $\mathbf{c}$ showing occipital nerves (Oc, red, orange and brown in d) exiting through occipital foramen (OcF) located anterior to the cranio-vertebral junction, and spinal nerves (Sp, black in d) exiting through vertebrae (V). (e) Embryonic alignment of caudal hindbrain, fourth ventricle, otic vesicle (OV) and myotomes (M) (includes blue lipophilic dye injection in fin buds; midshipman fish). The location of pectoral motoneurons is also indicated. NE, neuroepithelium. Images are dorsal $(\mathbf{a}, \mathbf{b}, \mathbf{e})$ and ventral (c, d) views with anterior to the left. Scale bars are $1 \mathrm{~cm}(\mathbf{a}), 200 \mu \mathrm{m}(\mathbf{b}), 500 \mu \mathrm{m}(\mathbf{c}, \mathbf{d})$ and $100 \mu \mathrm{m}(\mathbf{e})$. 
M2 (Fig. 2a-d). Location of the embryonic cranio-vertebral ${ }^{17}$ and hindbrain-spinal ${ }^{16}$ junctions between M3 and 4 showed the pectoral column to be in both the caudal hindbrain and rostral spinal cord with the fin innervated by Oc and Sp nerves (Fig. 2a-d; Supplementary Movie 1). Taken together, our results indicate that basal and derived actinopterygians share a conserved blueprint, with a hindbrainspinal pectoral motor nucleus innervating the fin via both Oc and Sp nerves.

Alignment with central and peripheral landmarks. Precise, threedimensional neuronal maps were obtained by aligning hindbrain nuclei with myotomes and peripheral nerves, complemented in transgenic zebrafish lines by genetic markers that label motoneuronal pools and demarcate the hindbrain-spinal boundary between M3 and 4. We first identified all hindbrain motoneurons exiting via Oc nerves by fluorescent labelling from the three most anterior myotomes, M1-3. In all cases, labelled occipital motoneurons formed a ventral column that began about one myotomal segment anterior to the pectoral column (Fig. 2e-h). The occipital column included all posterior hindbrain neurons projecting through Oc roots innervating pectoral fin, hypobranchial and axial (M1-3) muscles ${ }^{10}$ (summarized in Figure 2i). This overlapping occipital pectoral motoneuronal pattern further supported a generic blueprint of hindbrain-spinal pectoral innervation in actinopterygians.

We next aligned the pectoral motoneurons with other central nuclei using transgenic zebrafish lines. In the hoxb4a enhancer trap background (Figs $1 \mathrm{~b}$ and $3 \mathrm{a}$ ), a gata-2 promotor-driven YFP was inserted $3-\mathrm{kb}$ downstream of the endogenous hoxb4a gene $\mathrm{e}^{15}$. Many neurons from rhombomeres (r) 7-8 are genetically labelled with YFP, reflecting the presence of hoxb $4 a$ enhancer activity ${ }^{16}$ (Fig. 3a,b). The retrogradely labelled pectoral motor column was located in posterior $\mathrm{r} 8$ and extended into the adjacent spinal cord (Fig. 3c). Notably, the retrogradely labelled occipital motor column extended further rostral than pectoral motoneurons into the middle of $\mathrm{r} 8$ (Fig. 3d). Double labelling from the fin and cerebellum in an islet1-GFP transgenic background ${ }^{18}$ showed the hindbrain pectoral motoneurons to be located immediately caudal to precerebellar neurons (inferior olive/IO and area II/AII) in the middle of $\mathrm{r} 8^{16}$ (Fig. 3e-g; Supplementary Movie 2). A similar alignment of precerebellar and pectoral neurons in other teleosts studied (midshipman, trout and goldfish; Supplementary Fig. S4) strongly supported a common hindbrain neuronal map for actinopterygians. Occipital and pectoral motoneurons formed a single column $(\mathrm{Oc} / \mathrm{Pec})$ that was directly ventral to the posterior half of the vagal (X) motor nucleus in the caudal hindbrain and extended into the anterior spinal cord (Fig. 3h; also see Fig. 2e-h). Neuronal mapping with genetically and retrogradely labelled neurons thus showed pectoral motoneurons distributed across the hindbrain-spinal cord boundary and precisely positioned them with respect to other hindbrain subgroups (summarized in Figure 3i).

In situ origination of hindbrain pectoral motoneurons. The dual hindbrain-spinal origin of pectoral motoneurons was explicitly demonstrated using the photoconvertible protein kaede ${ }^{19}$ to trace the fate of the neuroepithelium in the hindbrain and anterior spinal cord, starting from early neurogenesis (Fig. 4a-e; Supplementary Movie 3). Kaede in both neuroepithelium and somites at the level of somites 4-5 was photoconverted from green to red at $12 \mathrm{~h}$ postfertilization (hpf; early neural keel stage $\mathrm{e}^{20}$; Fig. 4a,b) or at $18 \mathrm{hpf}$ (neural rod stage $\mathrm{e}^{20}$. Subsequent confocal analysis at 2 days postfertilization (dpf) showed that red kaede-labelled neurons remained at the level of M4-5 (Fig. 4c). Neurons located maximally 2-3 cell diameters away from the photoconverted area were occasionally observed (arrows, Fig. 4c). Retrograde labelling showed that pectoral motoneurons in the hindbrain at the level of M3 (Fig. 4c,d) did not contain the red converted version of kaede, unlike those in the anterior spinal cord (Fig. 4c,e). Pectoral motoneurons exhibited minimal, if any, anterior-posterior migration, regardless of whether the photoconversion was performed either at $12 \mathrm{hpf}(n=11)$ or $18 \mathrm{hpf}(n=10)$, or at the level of M3-4 $(n=2)$, M4-5 $(n=21)$ or M5-6 $(n=7)$.

Ontogeny of pectoral motoneurons. The location of pectoral motoneurons was further examined throughout early development
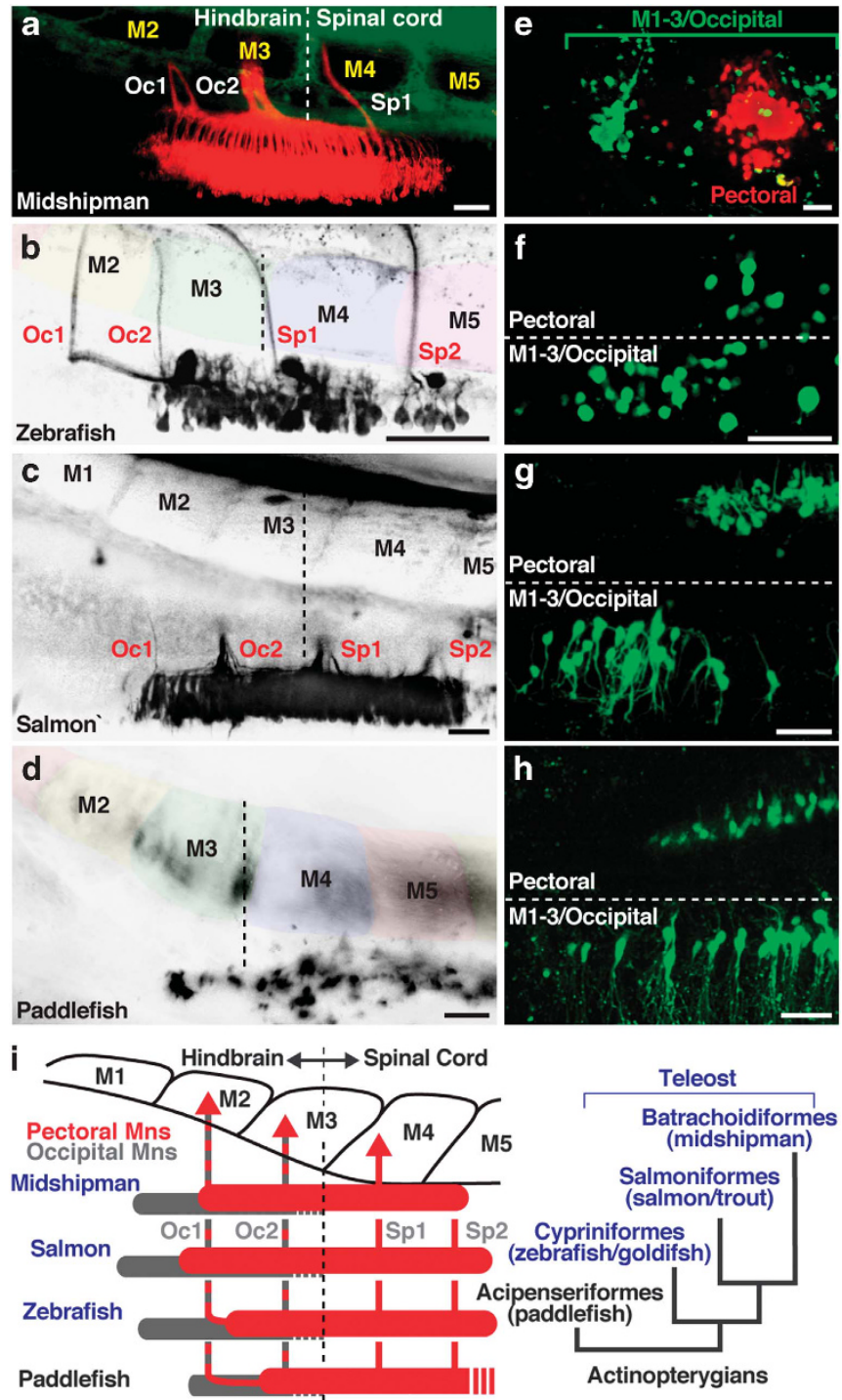

Figure 2 | Embryonic alignment of pectoral and occipital motoneurons with nerves and myotomes in basal and derived actinopterygians. (a-d) Location of pectoral motoneurons and nerves in actinopterygians revealed by lipophilic dye labelling from fin buds. The pectoral motor column began at the level of myotomes (M) 2-3 in all species studied (vertical hatching marks hindbrain-spinal boundary; also see Figure 1b,e). (e-h) Double labelling with fluorescent dextrans from fin buds and M1-3 showed that the occipital motor column began one myotomal segment anterior to pectoral motoneurons. Horizontal hatching marks midline in $\mathbf{f}-\mathbf{h}$. (i) Alignment of myotomes, nerves and motoneurons (pectoral/red and occipital/grey) with phylogenetic relationships of actinopterygians studied here (right). Paddlefish innervation pattern was deduced from juvenile gross anatomy (Supplementary Fig. S1-S2) as individual roots were not clearly visualized using retrograde labelling. All images are dorsal views with anterior to the left. Scale bars are $50 \mu \mathrm{m}$. Specimen stages: a (10 days postfertilization (dpf)/ $\sim 5.5 \mathrm{~mm}), \mathbf{b}(2 \mathrm{dpf} / \sim 3 \mathrm{~mm}), \mathbf{c}(100 \mathrm{dpf} / \sim 10 \mathrm{~mm}), \mathbf{d}(9 \mathrm{dpf} / \sim 13 \mathrm{~mm})$, $\mathbf{e}(18 \mathrm{dpf} / \sim 11 \mathrm{~mm}), \mathbf{f}(4 \mathrm{dpf} / \sim 4 \mathrm{~mm}), \mathbf{g}(115 \mathrm{dpf} / \sim 12 \mathrm{~mm}), \mathbf{h}(11 \mathrm{dpf} / \sim 16 \mathrm{~mm})$. 
by alignment in the hoxb4a enhancer trap background (Fig. 4f-k). During 2-20 dpf, when both pectoral musculature and innervation attain an adult configuration ${ }^{21,22}$, pectoral motoneurons increased in number in both hindbrain and spinal cord, splitting from a single motor pool into ventrolateral and dorsomedial columns (Fig. 4f,i). Their relative position to the hoxb4a-YFP domain indicated that pectoral motoneurons originated from, and were maintained, across the hindbrain-spinal cord boundary (Fig. $4 \mathrm{~g}, \mathrm{~h}, \mathrm{j}, \mathrm{k}$ ). On the basis of axonal trajectory, there was no evidence for pectoral motoneuron migration along the anteriorposterior axis (Fig. 4i-k). Taken together with the kaede experiments, these data demonstrated that pectoral motoneurons did not exhibit any longitudinal migration from early neurogenesis through
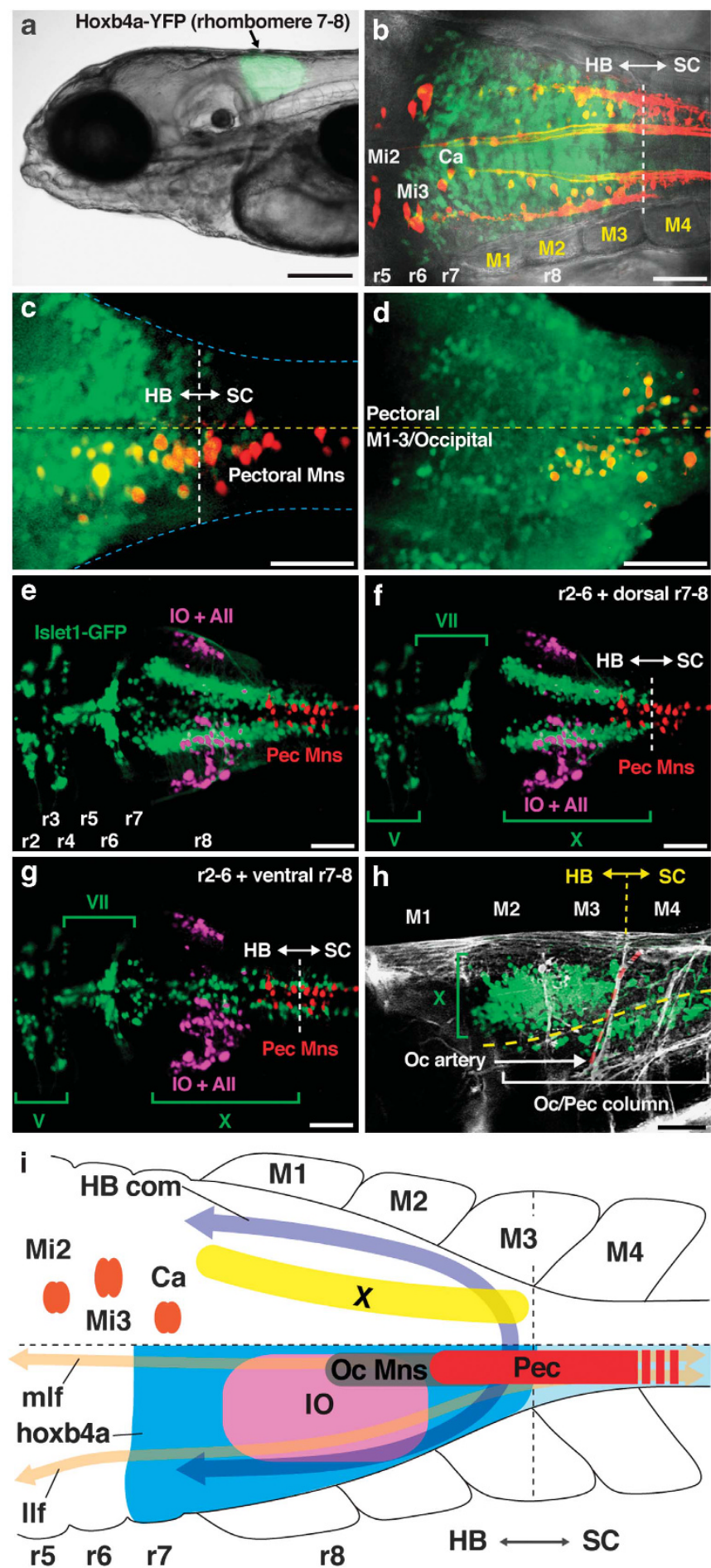

later development, similar to postmitotic spinal motoneurons in tetrapods ${ }^{23}$. Thus, pectoral motoneurons originated and remained across the hindbrain-spinal boundary in actinopterygian fish ${ }^{23}$.

Pectoral innervation in Chondrichthyes and Dipnoi. To facilitate comparisons with more readily available juvenile/adult sarcopterygian and the even more primitive cartilaginous fish/Chondrichthyes ${ }^{8}$, we investigated pectoral innervation in two lungfish/Dipnoi species (Protopterus dolloi and Lepidosiren paradoxa) and ratfish/ Chimaeriformes (Hydrolagus colliei). Ratfish had four Oc nerves (Oc1-4) that originated from the hindbrain as ventral roots directly behind the vagus nerve (X). All four nerve roots immediately combined into one nerve (Fig. 5a) that branched into hypobranchial and pectoral nerves at the level of the pectoral girdle (inset, Fig. 5a). The pectoral nerve immediately joined $S p$ nerves $1-3$ to form a plexus (inset, Fig. 5a) that innervated the pectoral fin along with 11 separate Sp nerve branches (Fig. 5a). Lungfish had three Oc nerves (Fig. 5b; Supplementary Fig. S5), with the most posterior root $(\mathrm{Oc} 3)$ emerging from the caudal hindbrain at a level near the end of the fourth ventricle (Fig. 5b). The Oc3 dorsal and ventral roots exited via two separate cranial foramina and fused into a single nerve immediately outside the skull (Supplementary Fig. S5a). Oc3 later merged with Sp1-3, forming a single pectoral nerve (Fig. 5; Supplementary Fig. S5). In summary, cartilaginous and sarcopterygian fish exhibited Oc and Sp innervation of the pectoral fin, similar to actinopterygians, supporting the hypothesis that a dual hindbrainspinal origin of pectoral motoneurons is an ancestral trait for jawed (cartilaginous and bony) vertebrates (summary, Fig. 6a).

\section{Discussion}

The conserved pectoral innervation pattern among jawed fish shown here, which consists of motoneurons and nerves originating from both the hindbrain and the spinal cord, differs notably from the spinal-only innervation in tetrapods. Our work in basal and derived actinopterygians supports the hypothesis that a dual hindbrainspinal origin for pectoral motoneurons in fish evolved into a spinalonly origin for forelimb motoneurons in tetrapods. This proposal is corroborated by new and previous evidence of pectoral innervation

Figure 3 | Embryonic alignment of precerebellar, pectoral and other hindbrain neurons in transgenic zebrafish. (a) Rhombomere ( $r$ ) 7-8 YFP expression in hoxb4a enhancer trap line. (b) Reticular (labelled from the spinal cord; red) and YFP (green) neurons showed hindbrain-spinal cord boundary between myotomes (M) 3-4. (c) Half of the pectoral column (labelled from fin bud; red) was within the hindbrain. (d) Occipital motor column (labelled from M1-3/occipital) extended to mid r8, one segment rostral to pectoral motoneurons. (e-g) Dorsal composites (e) and selected confocal planes $(\mathbf{f}, \mathbf{g})$ of pectoral (labelled from fin buds; red), inferior olive (IO) and Area II (AII) (labelled from the cerebellum; magenta) neurons in islet-GFP background (green), showing the relative position of pectoral motoneurons with major neuronal subgroups. GFP in this line is expressed in all hindbrain motoneurons, except abducens and pectoral. (h) Vagal (X) and more ventral occipital (Oc) motor columns that extended from spinal cord into the hindbrain (also see $\mathbf{f}, \mathbf{g}$ ). (i) Alignment of pectoral motoneurons $(\mathrm{Pec})$ with other neuronal and anatomical landmarks. Pectoral motoneurons in zebrafish were located across the hindbrain-spinal cord boundary at the level of M3-5 (see Figure 2). Hindbrain motoneurons are located immediately caudal to the inferior olive, below the vagal nucleus $(X)$ and hindbrain commissure (HB com). They are part of the occipital motor column (Oc Mns) at the level of two fibre tracts, the medial longitudinal fasciculus ( $\mathrm{mlf}$ ) and the lateral longitudinal fasciculus (IIf). Other abbreviations: Mi2, Mi3 and Ca, reticulospinal neurons ${ }^{53}$. Images are dorsal $(\mathbf{b}, \mathbf{c}, \mathbf{e}-\mathbf{g}, \mathbf{i})$, ventral $(\mathbf{d})$ and lateral $(\mathbf{a}, \mathbf{h})$ views with anterior to the left. Scale bars are $200 \mu \mathrm{m}$ (a), $50 \mu \mathrm{m}$ (b-h). Specimen stages: a, c, d (4dpf), b (2dpf), e-h (5dpf). 

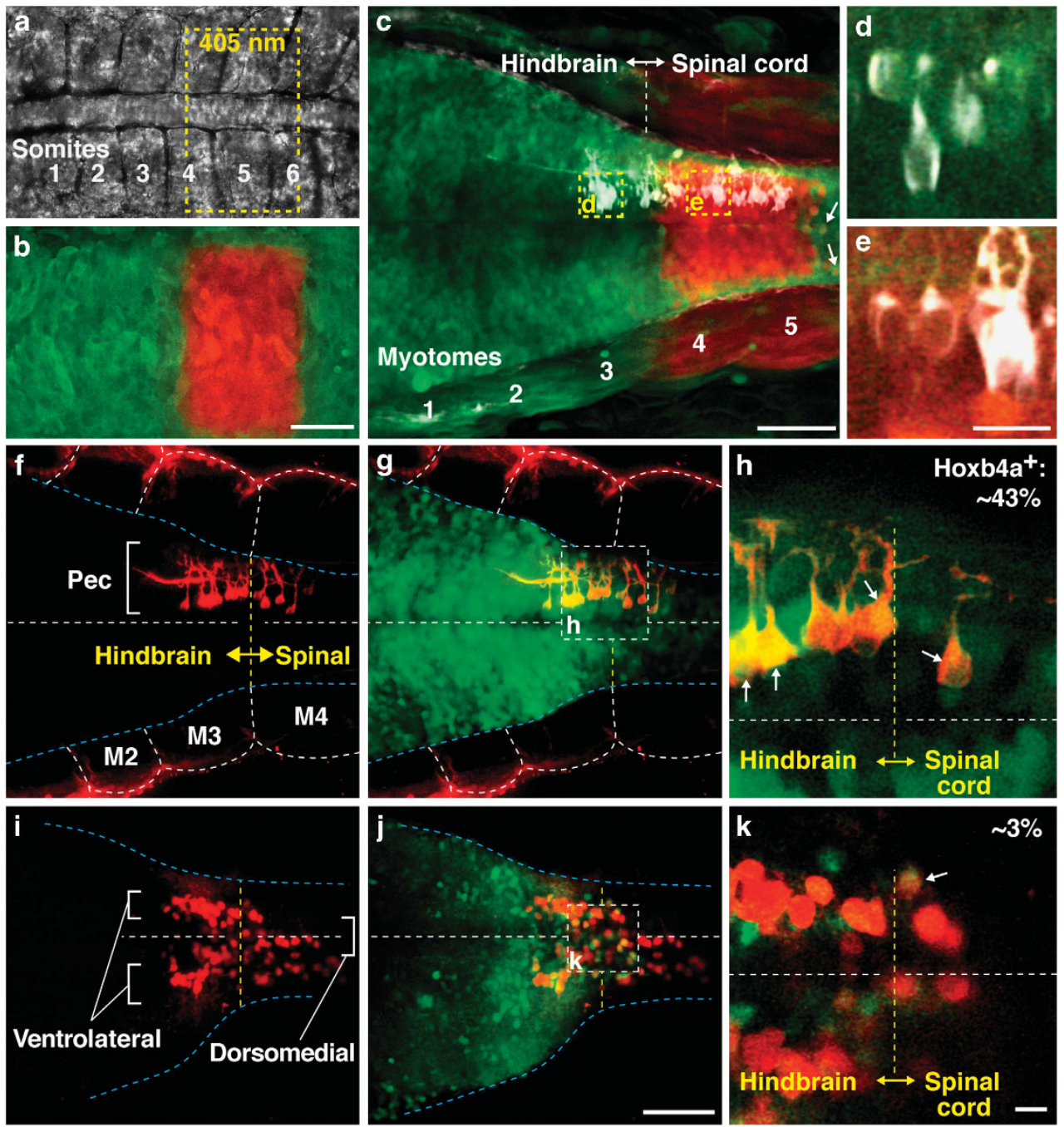

Figure 4 | Origin and maintenance of pectoral motoneurons across the hindbrain-spinal cord boundary. (a) Future hindbrain-spinal cord region in a $12 \mathrm{~h}$ postfertilization (hpf) embryo showing somites 1-6. (b) Transiently expressed kaede protein was photoconverted from green to red at the level of somites 4-5. Yellow dashed line in (a) marks the area of photoconversion using a laser of $405 \mathrm{~nm}$. (c) The same embryo at 2 days postfertilization (dpf) showed minimal, if any, anterior-posterior migration, with labelled neurons remaining tightly clustered at the level of myotomes (M) 4-5. Arrows point to neurons that were displaced 2-3 cell diameters posteriorly. (d, e) High magnification single plane images showed photoconverted kaede to be absent in pectoral motoneurons (white) at the level of M3 (d), but present in those at the level of M4 (e). (f-k) Ontogeny of pectoral motoneurons. At $2 \mathrm{dpf}$, pectoral motoneurons were labelled with lipophilic dye from fin bud (red) and appeared as a single column at the level of M3-4 (f) where they overlapped with the hoxb4a-YFP (green) expression domain ( $\mathbf{g}$, shown at a higher magnification in $\mathbf{h}$ ). At $20 \mathrm{dpf}$, pectoral motoneurons increased in number and developed into paired ventrolateral and dorsomedial columns (i). The motoneurons remained across the hindbrain-spinal cord boundary (yellow dashed line in $\mathbf{j}$ ) demarcated by YFP expression (shown at a higher magnification in $\mathbf{k}$ ) and exhibited a progressive downregulation of hoxb4a activity ${ }^{16}$ (arrows; $\mathbf{h}, \mathbf{k})$. Images are dorsal (a-h) and ventral (i-k) views with anterior to the left. Scale bars are $50 \mu \mathrm{m}(\mathbf{a}-\mathbf{c}, \mathbf{f}, \mathbf{g}, \mathbf{i}, \mathbf{j})$ and $10 \mu \mathrm{m}(\mathbf{d}, \mathbf{e}, \mathbf{h}, \mathbf{k})$. Specimen stages: a, b (12 hpf), c-h (2 dpf) and i-k (20 dpf).

from hindbrain/Oc and Sp nerves in lobe-finned and cartilaginous fish, implying that the pectoral motoneuron pool, similar to its target appendage, shifted caudally along the anterior-posterior axis during the fin-to-limb transition ${ }^{24}$.

Our embryonic data showed pectoral motoneurons located in the hindbrain of four actinopterygian species (Figs 2-4). Lineage tracing showed that these neurons originated in embryonic sites in both the hindbrain and spinal cord that were maintained throughout ontogeny in zebrafish (Figs 3 and 4). This embryonic blueprint was conserved among basal and derived actinopterygians (Fig. 2) and gave rise to adult innervation consisting of two Oc and various numbers (1-5) of Sp nerves (Figs 1 and 2; Supplementary Figs S1$\mathrm{S} 2$ ). We conclude that a dual hindbrain-spinal origin is the primitive trait for actinopterygian fish (Fig. 6a).
Sarcopterygians, the sister group of actinopterygians, also exhibited an occipital-spinal pectoral nerve pattern. As in the living coelacanth/Latimeria ${ }^{25,26}$, pectoral innervation in two species of lungfish/Dipnoi included an Oc nerve (Oc3) that originated from the hindbrain (Fig. 5; Supplementary Fig. S5). Similar to actinopterygians, the spinal contribution to pectoral innervation varied among sarcopterygians (11 in Latimeria versus 3 in P. dolloi). Together with our embryological results, we conclude that a hindbrain contribution to pectoral appendage innervation is the primitive condition for bony fish, including the sarcopterygians that gave rise to tetrapods (Fig. 6a).

We expanded our analysis to the most basal group of living jawed fish, Chondrichthyes, which include two subclasses, holocephalans such as the ratfish studied here and elasmobranchs 
(sharks and batoids). Pectoral innervation in ratfish, as in all other species studied here, involved Oc and Sp nerves (Fig. 5a). Hindbrain pectoral neurons were also reported in a batoid ${ }^{27}$, unlike the $\mathrm{Sp}$-only innervation reported in sharks ${ }^{28,29}$. We propose that $\mathrm{Oc} / \mathrm{Sp}$ pectoral innervation among actinopterygians, sarcopterygians and members of both subclasses of chondrichthyans (the elasmobranch pattern may be derived) represents the ancestral condition for all jawed vertebrates.
The general blueprint of a hindbrain-spinal motor column innervating occipital, hypobranchial and pectoral muscles (Figs $2 \mathrm{i}$ and $3 i$ ) is likely an ancestral feature linked to the original skull-fin musculoskeletal complex in fish (Fig. 6a). Fossil cephalaspids (jawless fishes/ostracoderms) exhibited well-developed pectoral fins that likely provided hydrodynamic lift, contributing to head orientation and sharp manoeuvres during swimming ${ }^{30}$. The fins were suggested to be innervated by the most anterior 2-3 ventral roots that emerged
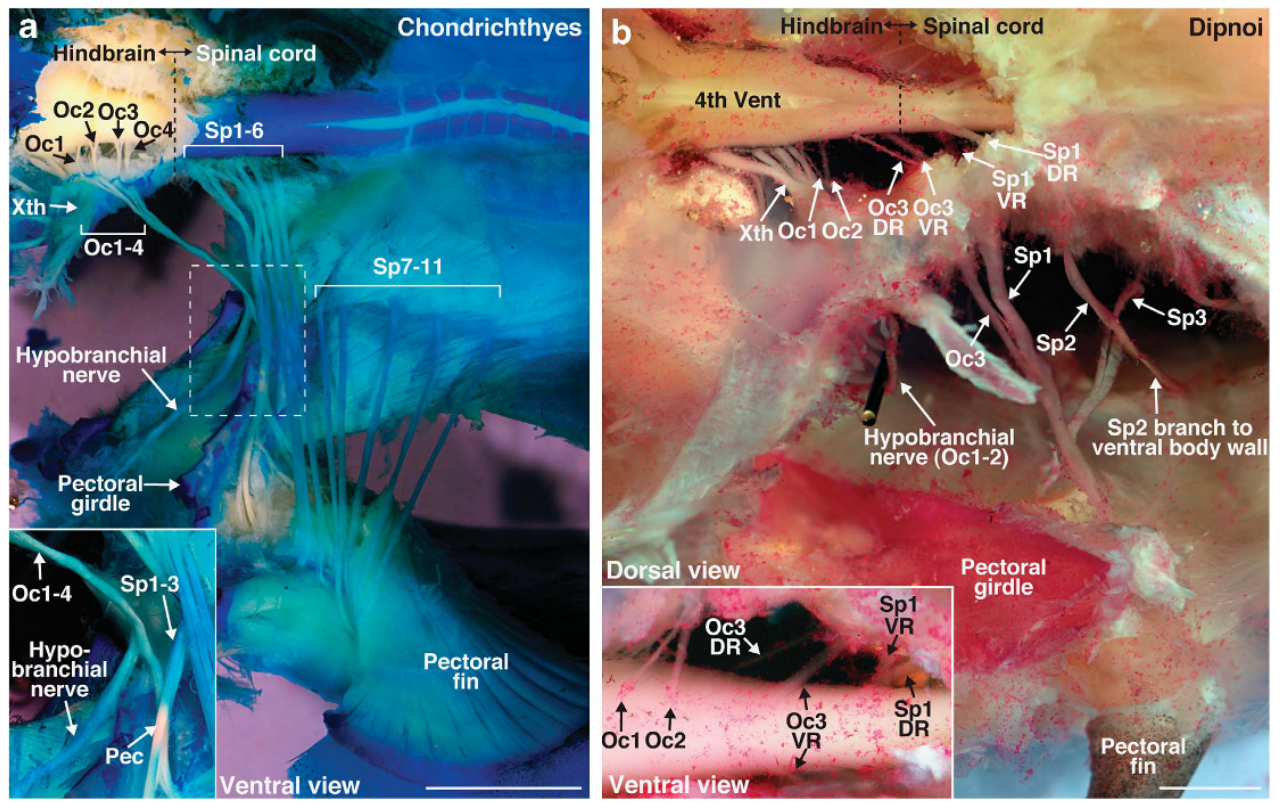

Figure 5 | Pectoral innervation in Chondricthyes (ratfish) and Dipnoi (lungfish). (a) Pectoral innervation in ratfish (H. colliei) included four occipital (Oc1-4) and 11 spinal nerves (Sp1-11). Inset shows the branching of Oc1-4 contributing to both hypobranchial nerve and the pectoral plexus. (b) Pectoral innervation in spotted African lungfish ( $P$. dolloi) included one occipital (Oc3) and three spinal nerves (Sp1-3). Oc3 dorsal (DR) and ventral (VR) roots emerged at the caudal end of the hindbrain. Inset shows the ventral view of Oc1-3 and Sp1. Scale bars are $5 \mathrm{~mm}$.

a
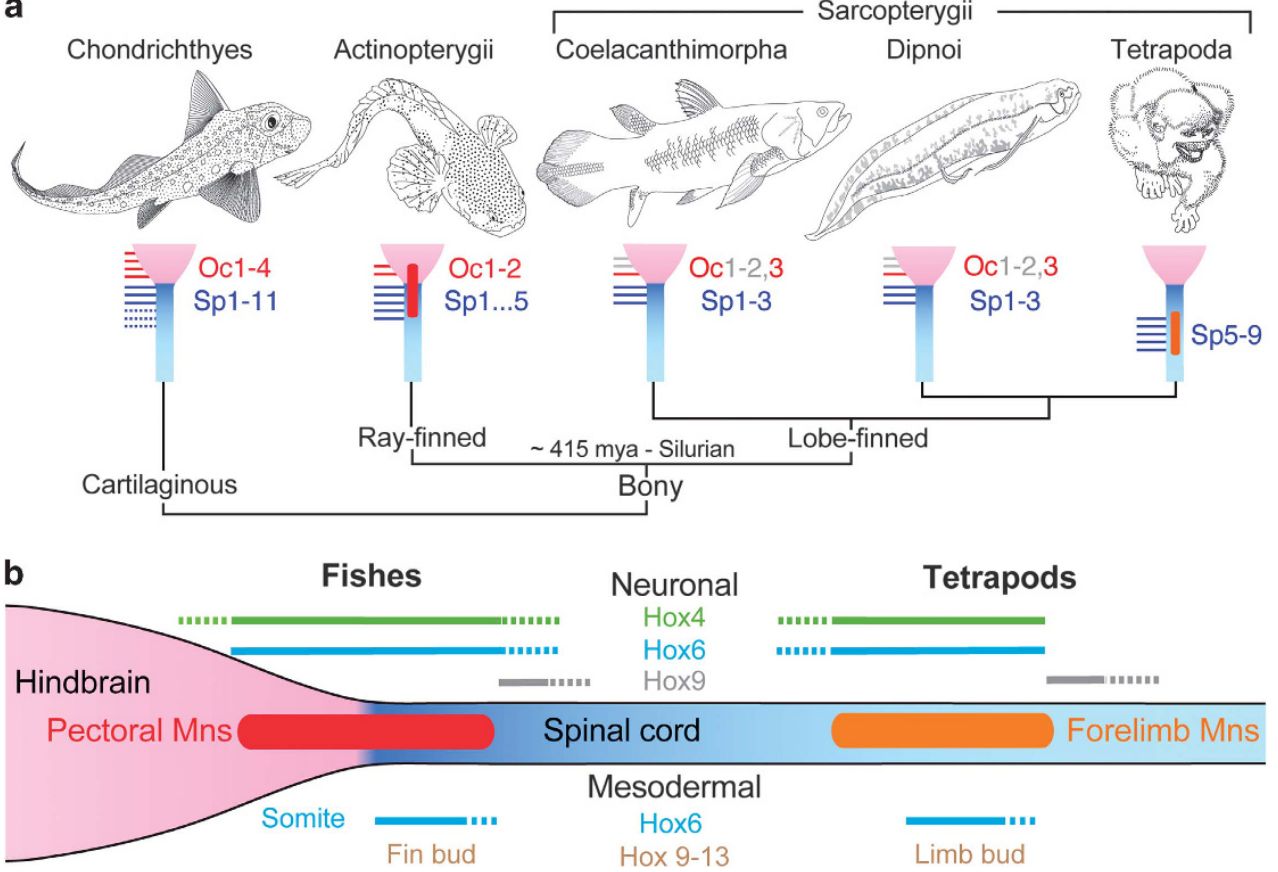

Figure 6 | Evolution of pectoral innervation. (a) Cladogram of living jawed vertebrates ${ }^{8}$, with vignettes showing innervation patterns of pectoral appendages. Occipital (pectoral, red; hypobranchial, grey) and spinal (blue) nerves are illustrated schematically. (b) Summary of key Hox genes expressed in neuronal (top) and mesodermal (bottom) compartments along the anterior-posterior axis in fish and tetrapods ${ }^{43,46,47,49,51}$. 
directly behind the vagus nerve ${ }^{31,32}$, similar to the condition in living jawed fish (Figs 1, 5 and 6; Supplementary Figs S1-S2, S5). The endoskeleton of cephalaspid pectoral fins was articulated to the cranium $^{33,34}$, analogous to the pectoral-occipital connection in early fossil sarcopterygians and primitive actinopterygians ${ }^{33,35}$ that share a similar pectoral endoskeletal structure ${ }^{36,37}$. The decoupling of this articulation and consequent increase in head mobility during the water-to-land transition, as seen in the fossil sarcopterygian fish Tiktaalik roseae $e^{2,38}$, may have been the reason for pectoral innervation being dissociated from the hindbrain. Unlike in fish, caudal hindbrain ventral roots in living amphibians contribute to $\mathrm{Sp}$ nerves 1-2 that innervate occipital, hypobranchial and hypoglossal muscles. Pectoral innervation typically arises from Sp nerves 3-5, with forelimb motoneurons located in the rostral spinal cord ${ }^{4,5}$. These observations in amphibians suggest that pectoral forelimb innervation in early tetrapods may have been shifted only a few segments caudally, relative to the pattern seen in sarcopterygian fish, despite separation of the skull and pectoral girdle ${ }^{38,39}$. In amniotes, there are generally six or more cervical vertebrae ${ }^{40}$ giving rise to a more elaborated neck structure. The rostral limit of pectoral forelimb innervation varies from Sp nerve 4 in some reptiles and mammals to Sp nerve 22 in long-necked birds ${ }^{6,41-44}$. As lower numbers of cervical vertebrae seem to be primitive for amniotes ${ }^{40}$, it seems that a caudal shift of about 5-6 segments in the location of the pectoral plexus, and therefore the motor nuclei, occurred between sarcopterygian fish and early amniotes (Fig. 6a). The absence of forelimb motoneurons in the tetrapod hindbrain can thus be viewed as the neuronal complement to the loss of the occipital-pectoral skeletal connection and the increased independence of head and body motion during the emergence of terrestrial vertebrates ${ }^{2,38}$.

The repositioning of both pectoral motoneurons and the pectoral girdle along the body axis likely depended, in part, on evolutionary developmental changes in the blueprint of Hox gene expression, which is important for both neuroectoderm and mesoderm patterning along the anterior-posterior axis ${ }^{45}$. The Hox regulatory framework is presumed to have instructed a repositioning of pectoral fin/forelimb in vertebrates ${ }^{43}$. The same mechanism likely positioned the motor column along with appendages at the corresponding axial level (Fig. 6b), as exemplified by Hox gene expression in both pectoral $^{16}$ and forelimb ${ }^{46}$ motoneurons. A comparison of Hox expression data from previous studies ${ }^{43,46-49}$ shows that fish and tetrapods share highly similar neuronal and mesodermal Hox codes for 'positioning' ${ }^{43}$ and 'patterning' ${ }^{36}$ the pectoral appendage and motoneuronal pool (Fig. 6b). Thus, the Hox code for both musculoskeletal and neuronal compartments is conserved for the pectoral motor system, despite anatomical differences in location along the anteriorposterior axis. As summarized in Figure $6 \mathrm{~b}$, we propose that the posterior shift of the pectoral motor nucleus involved sets of cisregulatory changes in Hox genes that led to both loss of pectoralrelated transcriptional machinery in the tetrapod hindbrain and correlated changes in the occipital, cervical and pectoral appendage peripheries $^{43,46,47,49-51}$.

In birds and mammals, Hox6 and Hox 9 paralogous groups confer, respectively, cervical and thoracic identity onto the neuroectoderm along the anterior-posterior axis ${ }^{46}$. In zebrafish, similar to birds and mammals, the anterior expression limits of Hox6/9 bracket the rostral-caudal extent of the pectoral motoneuron region ${ }^{48,49}$ (Fig. 6b). Therefore, the spatial relationship of Hox6/9 and pectoral motoneuron location has essentially been maintained during the caudal repositioning of the forelimb motor column (Fig. 6b). Similarly, in the mesoderm, the relative position of Hox6 expression and the axial level of limb/fin bud outgrowth seem to be conserved during pectoral appendage relocation between fish and tetrapods ${ }^{43}$ (Fig. 6b). Subsequent patterning of pectoral appendages also involves Hox9-13 triphasic expression in both zebrafish ${ }^{47}$ and tetrapods ${ }^{51}$.
After early regionalization of the neuroectoderm, Hox3-8 paralogous groups define forelimb motoneuron pools in birds and mammals ${ }^{46}$. The same set of Hox genes is expressed in the hindbrain-spinal region that gives rise to pectoral motoneurons in zebrafish ${ }^{48,49}$. Hence, we predict the presence of Hox3-8 in pectoral motoneurons, similar to their tetrapod counterparts, although only Hox4 has been directly demonstrated so far $^{16}$ (Fig. 6b). Conserved expression patterns in neuroectoderm, mesoderm and pectoral appendages are together suggestive of an ancestral neuronal/mesodermal Hox network linking motoneurons in both the hindbrain and spinal cord with the pectoral periphery (Fig. 6b). The evolutionary plasticity afforded by this Hox network provided a basis for further innovations in neural circuitry controlling forelimb functions essential to novel mechanisms of locomotion and feeding in tetrapods ${ }^{2,38}$.

\section{Methods}

Animals and specimens. Paddlefish were obtained from Osage Catfisheries (Osage Beach), salmon embryos from Grand Lake Stream Fish Hatchery, salmon juveniles and brook trout embryos from C Kraft and D Josephson (Department of Natural Resources, Cornell University, Ithaca, NY), goldfish from Hunting Greek Fisheries, midshipman from M Marchaterre (Department of Neurobiology and Behavior, Cornell University, Ithaca, NY), fixed specimens of ratfish from J Sisneros (Department of Psychology, University of Washington, Seattle, WA, USA) and South American and South African lungfishes from Tropical Fish Distributors. Animal handling and experimental procedures were approved by the Institutional Animal Care and Use Committee of New York University School of Medicine (New York, NY, USA), Cornell University (Ithaca, NY, USA) and Marine Biological Laboratory (Woods Hole, MA, USA).

Fixation and staining for gross anatomy. Juveniles/adults were killed by an overdose of ethyl 3-aminobenzoate methanesulphonate (MS 222, Sigma), fixed by $4 \%$ paraformaldehyde perfusion and then stained sequentially with alcian blue and alizarin red before dissection ${ }^{52}$. For some paddlefish, nerves were stained briefly with $2 \%$ osmium tetroxide solution after fixation.

Retrograde labelling. Embryos and larvae were anaesthetized with $0.02 \% \mathrm{MS}$ 222 and immobilized in $2 \%$ low-gelling agarose for imaging ${ }^{16}$. For retrograde labelling in early embryos, $50-100 \mathrm{nl}$ of saturated mixture of the lipophilic dye $\mathrm{DiD}$ (Invitrogen) in 50\% vegetable oil/50\% dimethylsulphoxide was pressure injected into the fin bud at $40 \mathrm{psi}$. As lipophilic dye works very poorly in live specimens later in development, pectoral motoneurons in larvae were labelled by injecting 200-300 $\mathrm{nl}$ of Alexa Fluor 647 dextran (3\% in $0.2 \mathrm{KCl}$ with $0.1 \%$ Triton $\mathrm{X}-100$ ) into the fin musculature. Double labelling of either Oc or precerebellar neurons was achieved by additional dye injection into, respectively, M1-3 $(\sim 200 \mathrm{nl}$ of either Alexa Flour 488 or 647 dextran; $3 \%$ in $0.2 \mathrm{KCl}$ with $0.1 \%$ Triton $\mathrm{X}-100)$ or the cerebellum ( $\sim 50 \mathrm{nl}$ of either Alexa Fluor 488 or 594 dextran; 3\% in $0.2 \mathrm{KCl}$ with $0.1 \%$ Triton $\mathrm{X}-100)^{16}$.

Kaede lineage tracing. Lineage tracing experiments were conducted by injecting 500 pg of mRNA encoding the photoconvertable protein kaede into single-cellstage zebrafish embryos ${ }^{19}$. Neuroepithelium and somites at the level of somites 4-5 were scanned by a laser of $405 \mathrm{~nm}$ on a confocal microscope at either 12 or $18 \mathrm{hpf}$, which induced green-to-red photoconversion of cytosolic kaede protein Lineage of cells containing converted kaede was examined using a confocal microscope at $2 \mathrm{dpf}$.

Confocal images were obtained using a Zeiss 510 system and processed using Image (NIH) and Photoshop (Adobe).

\section{References}

1. Pough, F. H., Janis, C. M. \& Heiser, J. B. Vertebrate Life 8th edn (Benjamin Cummings, 2009).

2. Shubin, N. H., Daeschler, E. B. \& Jenkins, F. A. Jr. The pectoral fin of Tiktaalik roseae and the origin of the tetrapod limb. Nature 440, 764-771 (2006)

3. Shubin, N., Tabin, C. \& Carroll, S. Deep homology and the origins of evolutionary novelty. Nature 457, 818-823 (2009).

4. Stephens, N. \& Holder, N. A horseradish peroxidase study of motorneuron pools of the forelimb and hindlimb musculature of the axolotl. Proc. R Soc. Lond B Biol. Sci. 224, 325-339 (1985).

5. Oka, Y., Ohtani, R., Satou, M. \& Ueda, K. Location of forelimb motoneurons in the Japanese toad (Bufo japonicus): a horseradish peroxidase study. J. Comp. Neurol. 286, 376-383 (1989).

6. Ryan, J.M. et al. Topographic position of forelimb motoneuron pools is conserved in vertebrate evolution. Brain Behav. Evol. 51, 90-99 (1998). 
7. Uemura, O. et al. Comparative functional genomics revealed conservation and diversification of three enhancers of the isl 1 gene for motor and sensory neuron-specific expression. Dev. Biol. 278, 587-606 (2005).

8. Nelson, J. S. Fishes of the World (John Wiley \& Sons, 2006).

9. Allis, E. P. J. The skull, and the cranial and first spinal muscles and nerves in Scomber scomber. J. Morphol. 18, 45-328 (1903).

10. Winterbottom, R. A descriptive synonmy of the striated muscles of the Teleosteii. Proc. Acad. Natl Sci. Phil. 125, 225-317 (1973).

11. Parenti, L. \& Song, J. in Interrelationships of Fishes (eds Stiassny, M. L. J., Parenti, L. R., Johnson, G. D.) Ch. 16, 472-444 (Academic Press, 1996).

12. Braus, H. Ueber die Innervation der paarigen Extremitäten bei Selachiern, Holocephalen und Dipnoern. Jena Zeitschr. Naturwiss. 31, 239-486 (1898).

13. Bemis, W. E. \& Forey, P. L. in Major Events in Early Vertebrate Evolution Paleontology, Phylogeny, Genetics and Development (ed. Ahlberg, P.) 350-369 (Taylor and Francis, 2000)

14. Hara, T. \& Zielinski, B. Sensory Systems Neuroscience (Elsevier, 2006).

15. Punnamoottil, B. et al. Enhancer detection in zebrafish permits the identification of neuronal subtypes that express Hox4 paralogs. Dev. Dyn. 237, 2195-2208 (2008)

16. Ma, L. H., Punnamoottil, B., Rinkwitz, S. \& Baker, R. Mosaic hoxb4a neuronal pleiotropism in zebrafish caudal hindbrain. PLoS One 4, e5944 (2009).

17. Morin-Kensicki, E. M., Melancon, E. \& Eisen, J. S. Segmental relationship between somites and vertebral column in zebrafish. Development 129, 3851-3860 (2002)

18. Higashijima, S., Hotta, Y. \& Okamoto, H. Visualization of cranial motor neurons in live transgenic zebrafish expressing green fluorescent protein under the control of the islet-1 promoter/enhancer. J. Neurosci. 20, 206-218 (2000)

19. Ando, R., Hama, H., Yamamoto-Hino, M., Mizuno, H. \& Miyawaki, A. An optical marker based on the UV-induced green-to-red photoconversion of a fluorescent protein. Proc. Natl Acad. Sci. USA 99, 12651-12656 (2002).

20. Tawk, M. et al. A mirror-symmetric cell division that orchestrates neuroepithelial morphogenesis. Nature 446, 797-800 (2007).

21. Thorsen, D. H. \& Hale, M. E. Development of zebrafish (Danio rerio) pectoral fin musculature. J. Morphol. 266, 241-255 (2005).

22. Thorsen, D. H. \& Hale, M. E. Neural development of the zebrafish (Danio rerio) pectoral fin. J. Comp. Neurol. 504, 168-184 (2007).

23. Leber, S. M. \& Sanes, J. R. Migratory paths of neurons and glia in the embryonic chick spinal cord. J. Neurosci. 15, 1236-1248 (1995).

24. Goodrich, E. S. Studies on the Structure and Development of Vertebrates (Macmillan, 1930)

25. Millot, J. \& Anthony, J. Anatomie de Latimeria Chalumnae: Système nerveux et organes des sens Vol 2 (Centre national de la recherche scientifique, 1965).

26. Northcutt, R. G. \& Bemis, W. E. Cranial nerves of the coelacanth, Latimeria Chalumnae [Osteichthyes: Sarcopterygii: Actinistia], and comparisons with other craniata. Brain Behav. Evol. 42(Suppl 1), 1-76 (1993).

27. Sperry, D. G. \& Boord, R. L. Organization of the hypobranchial motor column of the clearnose skate, Raja eglanteria, with comparisons to tetrapods. Acta. Anat. (Basel) 160, 21-32 (1997).

28. Müller, E. Untersuchungen über die Muskeln und Nerven der Brustflosse und der Körperwand bei Acanthias vulgaris. Anatomy and Embryology 43, 1-147 (1911).

29. Norris, H. W. \& Hughes, S. P. The cranial, occipital, and anterior spinal nerves of the dogfish, Squalus acanthias. J. Comp Neurol 31, 293-402 (1920).

30. Bunker, S. J. \& Machin, K. E. The hydrodynamics of cephalaspids. Soc. Exp. Biol. Seminar Ser. 36, 113-129 (1991).

31. Janvier, P. Les Cephalaspides Du Spitsberg: Anatomie, Phylogenie Et Systematique Des Osteostraces Siluro-Devoniens, Revision Des Osteotraces De La Formation De Wood Bay (Devonien Inferieur Du Spitsberg) (Editions du Centre national de la recherche scientifique, 1985).

32. Janvier, P. Les nageoires paires des Ostéostracés et la position systématique des Céphalaspidomorphes. Annales de Paléontologie 64, 113-142 (1987).

33. Janvier, P. Early Vertebrates (Clarendon Press, 1996).

34. Janvier, P., Arsenault, M. \& Desbiens, S. Calcified cartilage in the paired fins of the osteostracan Escuminaspis laticeps (Traquair 1880), from the Late Devonian of Miguasha (Québec, Canada), with a consideration of the early evolution of the pectoral fin endoskeleton in vertebrates. J. Vert. Paleo. 24, 773-779 (2004)

35. Davis, M. C., Shubin, N. H. \& Force, A. Pectoral fin and girdle development in the basal actinopterygians Polyodon spathula and Acipenser transmontanus. J. Morphol. 262, 608-628 (2004).
36. Friedman, M., Coates, M. I. \& Anderson, P. First discovery of a primitive coelacanth fin fills a major gap in the evolution of lobed fins and limbs. Evol. Dev. 9, 329-337 (2007)

37. Mabee, P. M. \& Noordsy, M. Development of the paired fins in the paddlefish, Polyodon spathula. J. Morphol. 261, 334-344 (2004).

38. Daeschler, E. B., Shubin, N. H. \& Jenkins, F. A. Jr. A Devonian tetrapod-like fish and the evolution of the tetrapod body plan. Nature 440, 757-763 (2006).

39. de Beer, G. R. The Development of the Vertebrate Skull (Oxford University Press, 1937).

40. Muller, J. et al. Homeotic effects, somitogenesis and the evolution of vertebral numbers in recent and fossil amniotes. Proc. Natl Acad. Sci. USA 107, 2118-2123 (2010).

41. Koizumi, M. \& Sakai, T. On the morphology of the brachial plexus of the platypus (Ornithorhynchus anatinus) and the echidna (Tachyglossus aculeatus). J. Anat. 190 (Part 3), 447-455 (1997).

42. Howell, A. B. Morphogenesis of the shoulder architecture: Aves. Auk 54, 364-375 (1937).

43. Burke, A. C., Nelson, C. E., Morgan, B. A. \& Tabin, C. Hox genes and the evolution of vertebrate axial morphology. Development 121, 333-346 (1995).

44. Bubien-Waluszewska, A. in Form and Function in Birds Vol. 3 (eds King, A.S. McLelland, J.) 149-194 (Academic Press, 1985).

45. Pearson, J. C., Lemons, D. \& McGinnis, W. Modulating Hox gene function during animal body patterning. Nat. Rev. Genet. 6, 893-904 (2005).

46. Dasen, J. S. \& Jessell, T. M. Hox networks and the origins of motor neuron diversity. Curr. Top Dev. Biol. 88, 169-200 (2009).

47. Ahn, D. \& Ho, R. K. Tri-phasic expression of posterior Hox genes during development of pectoral fins in zebrafish: implications for the evolution of vertebrate paired appendages. Dev. Biol. 322, 220-233 (2008).

48. Prince, V. E., Joly, L., Ekker, M. \& Ho, R. K. Zebrafish hox genes: genomic organization and modified colinear expression patterns in the trunk. Development 125, 407-420 (1998)

49. Prince, V. E., Moens, C. B., Kimmel, C. B. \& Ho, R. K. Zebrafish hox genes: expression in the hindbrain region of wild-type and mutants of the segmentation gene, valentino. Development 125, 393-406 (1998)

50. Wagner, G. P. The developmental genetics of homology. Nat. Rev. Genet. 8, 473-479 (2007).

51. Zakany, J. \& Duboule, D. The role of Hox genes during vertebrate limb development. Curr. Opin. Genet. Dev. 17, 359-366 (2007).

52. Song, J. \& Parenti, L. R. Clearing and Staining Whole Fish Specimens for Simultaneous Demonstration of Bone, Cartilage, and Nerves. Copeia 1995 114-118 (1995)

53. Trevarrow, B., Marks, D. L. \& Kimmel, C. B. Organization of hindbrain segments in the zebrafish embryo. Neuron 4, 669-679 (1990).

\section{Acknowledgments}

We thank A. Rice for skeletal preparation in Figure 1a; M. Nelson for drawings in Figure 6a; C. Kraft, D. Josephson and M. Marchaterre for field assistance with embryo collections; and H. Baker, J. Bassett, W. Bemis, B. Chagnaud, J. Maisey, A. Pastor, F. Rijli and K. Zamudio for discussion and comments on early versions of the paper. This work was supported by the National Institutes of Health and National Science Foundation.

\section{Author contributions}

L.M. and E.G. conducted the experiments. All authors designed the experiments, analysed the data and wrote the paper.

\section{Additional information}

Supplementary Information accompanies this paper on http://www.nature.com/ naturecommunications

Competing financial interests: The authors declare no competing financial interests.

Reprints and permission information is available online at http://npg.nature.com/ reprintsandpermissions/

How to cite this article: Ma, L.-H. et al. Ancestry of motor innervation to pectoral fin and forelimb. Nat. Commun. 1:49 doi: 10.1038/ncomms1045 (2010).

Licence: This work is licensed under a Creative Commons Attribution-NonCommercial-No Derivative Works 3.0 Unported License. To view a copy of this license, visit http:// creativecommons.org/licenses/by-nc-nd/3.0/ 